\title{
UN ANÁLISIS DE LA VIOLENCIA Y EL SEXISMO DESDE EL IMAGINARIO MUSICAL ECUATORIANO DE LA REGIÓN COSTA ${ }^{1}$
}

Cristina Mateos Casado². Universidad Técnica de Manabí. Ecuador. cristina mateos@hotmail.com cmateos@utm.edu.ec

María Pita Asan: Universidad Técnica de Manabí. Ecuador. mpita@utm.edu.ec

Mónica Vélez Zambrano: Universidad Técnica de Manabí. Ecuador. moliveza@hotmail.com

Robert Cedeño Mejía: Universidad Técnica de Manabí. Ecuador. robsinger-gk@hotmail.com

José Ruíz Villamar: Universidad Técnica de Manabí. Ecuador. eduruvill@hotmail.com

\section{RESUMEN:}

La socialización de los géneros se da en un proceso de aprendizaje cultural de los papeles asignados según el sexo, identificándose de forma transversal en todas las culturas. Las normas de género y la construcción de la feminidad y la masculinidad conforman un mundo binario generador de desigualdades. De entre todas las instituciones y elementos de socialización hemos elegido, a través de una metodología de Investigación Acción Participativa (IAP) en el aula, el análisis de la violencia y el sexismo desde la música, ya que ésta constituye en todo el mundo un fenómeno de masas que contribuye y perpetúa fuertes imaginarios sociales y culturales discriminatorios. En este proceso de análisis, las alumnas y los alumnos han formado parte de la muestra y también han participado en el desarrollado de la primera fase de la investigación. Para ello, se ha realizado un análisis de los gustos musicales desde una perspectiva de género que ha permitido extraer conclusiones respecto al sexismo y la construcción del imaginario de amor romántico. Para

\footnotetext{
${ }^{1}$ Las Regiones Naturales del Ecuador son: Costa, Sierra, Oriente y Galápagos. La Región Costa se dividida en siete provincias: Esmeraldas, Santo Domingo de los Tsáchilas, Manabí, Guayas, Santa Elena, Los Ríos y El Oro. El estudio se ha realizado en el Cantón Portoviejo, capital de Manabí.

2 Cristina Mateos Casado: Trabajadora Social, Socióloga y Doctorado en Feminismo por la Universidad de Salamanca y la Universidad Complutense de Madrid, respectivamente. En la actualidad se desempeña como docente investigadora a tiempo completo en la Universidad Técnica de Manabí, Ecuador.
}

Correo: cristina mateos@hotmail.com cmateos@utm.edu.ec 
finalizar, se ha contrastado la muestra de las alumnas y los alumnos que habían cursado la asignatura Perspectiva de género o Equidad de género con las alumnas y los alumnos que no habían cursado dichas asignaturas para determinar en qué medida la adquisición de la conciencia social de género influye en el imaginario musical o, si por el contrario, este tipo de imaginario se conforma en el proceso de socialización a edades muy tempranas y es difícil su modificación a través de la adquisición reciente de nociones de género.

PALABRAS CLAVE: Perspectiva de género - Imaginario musical - Género musical Canción - Identidad cultural - Violencia - Sexismo.

\section{ANALYSIS OF VIOLENCE AND SEXISM FROM THE MUSICAL ECUADORIAN IMAGINARINESS OF THE COSTA REGION}

\section{ABSTRACT:}

Socialization of genders occurs in a process of cultural learning of the roles assigned according to sex. It is transversally identified in all cultures. Gender standards and the construction of femininity and masculinity make an inequality-generating binary world. Through a methodology of participative action research, we have chosen the analysis of violence and sexism in music from all institutions and elements of socialization. Because music is a phenomenon of masses around the world which contributes to and keeps strong discriminatory social and cultural imaginariness. In this process of analysis, students have been part of the sample data and they have participated in the development of the first stage of research. First, we have made an analysis of the musical likes from a gender perspective which has allowed us to come to some conclusions regarding sexism and the construction of romantic love imaginariness. Finally, we have contrasted the sample of the students that studied the subject Gender Perspective or Gender Equity with those who did not take that subject to determine how the acquisition of social awareness of gender influences musical imaginariness or if, on the contrary, this kind of imaginariness is made up in the process of socialization at very early ages and it is difficult to modify it through the acquisition of recent notions of gender.

KEY WORDS: Gender perspective - Musical imaginariness - Musical style - Song Cultural identity - Violence - Sexism. 


\section{INTRODUCCIÓN}

\subsection{La violencia y el sexismo como espectáculo. "Sharon, el femicidio de la cantante que conmovió al país" ${ }^{3}$}

En Ecuador, 6 de cada 10 mujeres sufren algún tipo de violencia según la "Encuesta Nacional de relaciones familiares y violencia de género contra las mujeres" (INEC, 2012), que fue la primera encuesta de este tipo publicada en Ecuador e impulsada por la Comisión de Transición hacia el Consejo de las Mujeres y la Igualdad de Género, bajo la dirección de Ana Lucía Herrera ${ }^{4}$.

El delito de femicidio se incorporó en la reforma del Código Orgánico Integral Penal (COIP) en el año 2014. Posiblemente, esta figura jurídica no se dio a conocer en el país hasta el "presunto" ${ }^{5}$ asesinato por violencia de género de la cantante más popular de la Tecnocumbia ecuatoriana, Edith Rosario Bermeo Cisneros, artísticamente conocida como Sharon La Hechicera.

Una multitud de personas despidieron en enero del año 2015 (El Comercio, 6 de enero de 2015) a la cantante bajo el sensacionalismo propio de una cultura y una sociedad que han convertido la muerte en espectáculo. Sería una muerta más para lo que Marzano (2010) denomina "muertes para la pantalla", en donde no se sabe dónde acaba el horror por la muerte y empieza el horror por convertir la muerte en una mercancía de consumo que se vende, se negocia, se comenta, se comparten fotos y videos de su muerte, se twittean las opiniones de su hija o las frases del hijo menor que la acompañaba en el supuesto asesinato.

Una muerte tolerada socialmente. La revictimización de la mujer, aún muerta, es evidente, bajo una "pedagogía de la crueldad" como señala la antropóloga argentina Rita Segato: "El público es enseñado a no tener empatía con la víctima, que es revictimizada con banalidad y espectacularización" (Entrevista en la Universidad de Costa Rica, 2015).

Ecuador fue un país pionero en América Latina en conseguir el derecho a voto para las mujeres en el año 1929. En décadas posteriores, los Movimientos Sociales y el Movimiento de Mujeres (MM) han sido activos en la Región en la defensa de los derechos sociales y de género. Sin embargo, como otros muchos países, las cifras de la desigualdad son alarmantes: 6 de cada 10 mujeres han sufrido violencia de género (INEC, 2012); 1 de cada 4 mujeres ha sufrido violencia sexual, en su mayoría en el

\footnotetext{
${ }^{3}$ Hace referencia al titular elegido por el periódico El Comercio al cumplirse dos meses del supuesto femicidio de la artista. "Sharon, un caso que conmovió al país". (El Comercio, 25 de Febrero de 2015) Recuperado de http://www.elcomercio.com/actualidad/sharon-fallecimiento-cronologia-cantante.html

${ }^{4}$ Ana Lucía Herrera, feminista ecuatoriana de enorme influencia para los avances en igualdad de género en Ecuador. Presidenta de la Comisión de transición hacia el consejo de las mujeres y la igualdad de género desde el año 2009 al 2012. En el año 2014 la Comisión se transformó en Consejo nacional para la igualdad de género.

5 Se nombra como "presunto asesino" ya que desde febrero de 2015 la única noticia que ha trascendido es que se acogió el dictamen acusatorio emitido por la Fiscalía y llamó a juicio a Geovanny L., por el delito de femicidio en grado de tentativa. En un primer momento la muerte de la cantante se vinculó a un accidente de tránsito.
} 
ámbito intrafamiliar (INEC, 2012); el embarazo preadolescente, entre diez y catorce años, aumentó en un $78 \%$ en los últimos diez años (INEC, 2012); y las tres primeras causas de suicidio en mujeres jóvenes de diez a catorce años son las depresiones causadas por violencia, embarazos precoces y relaciones amorosas (CNNA).

Pero estos datos no escandalizan en exceso. Tampoco que los restos mortales de $\mathrm{La}$ Hechicera fueran despedidos en un ambiente festivo con su música sonando desde los altavoces transportados en varios vehículos, en un cortejo que recorrió por más de dos horas las calles de su ciudad natal, Durán, en donde las personas desde los balcones ovacionaban su figura ajenas a la tragedia de su muerte. Toda la representación se resume en una idea "tranquilizadora" que se repite: "muere la artista y nace la leyenda" (E/ Comercio, 6 de enero de 2015). Una leyenda que presidía el reinado de un fenómeno "para el pueblo" como es la Tecnocumbia, reafirmando así que se trata efectivamente de un fenómeno que se enmarca principalmente en una estética de las clases populares (Santillán y Ramírez, 2004: p. 44)

La tragedia es seguida críticamente desde el feminismo ecuatoriano. La activista María Belén Moncayo denuncia la invisibilidad de otros femicidios en el país y plantea una responsabilidad de Estado:

¿Debieron entonces Vanessa Landines Ortega, Angélica Balladares y todas las mujeres ecuatorianas que han sido muertas por femicidio, ser famosas, guapas y sexys para que entonces desde el Ministerio del Interior empiecen a sentir afecto en tanto seres humanos y por ende atender con diligencia y verdadera justicia sus casos particulares de asesinato?. (Perfil de Facebook, 5 de enero de 2015)

La vida y la muerte de Sharon se transformaron en consumo cultural, entendiendo ésta noción como "una práctica socialmente condicionada en la que los individuos actúan selectivamente al apropiarse y hacer suyos determinados bienes y prácticas, dándoles usos y sentidos sociales locales" (Santillán y Ramírez, 2004: p. 46). No sabemos dónde empieza y termina el producto cultural, la muerte real y la muerte ficcionada de la cantante, ya que su último videoclip se toma como "presagio":

El video del tema "Madrecita por qué me dejas" muestra al hijo menor de Sharon, de casi 3 años, echando al mar un alijo mortuorio con flores y la foto de la propia Sharon. En otra escena, la cantante camina por una carretera cercana al mar y luego desaparece. Adiós, hijitos, les voy dejando, al fin reciban mi bendición, se escucha en una parte del video, que termina con un susurro de la artista dirigido presumiblemente a su hijo: Te prometo, allá en el cielo te veo. (El Comercio, 6 de enero de 2015)

La música es un reflejo de la sociedad. Sociológicamente se ha definido la llamada "sociedad narco", fomentada por una "narco cultura" y una "narco estética" que guarda una profunda relación con el sexismo y la violencia de género. En el caso de México, existe toda una industria que produce "narcocorridos", "narcopelículas" y "narcoliteratura". Los "narcocorridos" son composiciones musicales en los que se recoge el mundo del narcotráfico y de los narcotraficantes. En sus narrativas dan 
cuenta de la violencia, el poder, la muerte, el consumo excesivo y los supuestos "placeres" derivados de las acciones del narcotráfico.

Ecuador no es ajeno a tal realidad. La Oficina de Naciones Unidas contra la Droga y el Delito (UNODC) indica que el país geográficamente se encuentra en medio de la "ruta de la cocaína", siendo América (prioritariamente Estados Unidos), Europa y Oceanía los principales mercados según el "Informe mundial sobre drogas 2012". Los cárteles de la droga han extendido sus negocios también por Ecuador, especialmente el cartel de Sinaloa, con repercusiones en la cultura y en la identidad nacional. La influencia de series de televisión como "Sin tetas no hay paraíso" o las "Muñecas de la mafia" han logrado de alguna manera que la población se identifique con ellas y con la supuesta "sociedad del éxito" que representan. No sólo las historias y la imagen, también la música es fundamental para la identificación y reproducción de un modelo social basado en las desigualdades y la violencia:

Quiero ser la reina pa' cuando vuelva/ por eso quiero un hombre rico pa' que me mantenga/ dile a todo el mundo que estás con una hembra/ que tienes un juguete nuevo pa' que te entretenga/ todo lo que se consigue con una mini usi, una finca grande con jardín y jacuzzi/ méteme al certamen de reina de belleza, no puedes pedir tanto con tan poco en la cabeza (Canción de la serie de televisión "Las muñecas de la mafia").

En Ecuador, en la provincia de Manabí, se desarrolla en los años 90 un tipo de cine de alto contenido violento, denominado "Cine Chonero", en referencia a la banda de delincuencia organizada Los Choneros. La película "Sicarios manabitas" (2004), del director Fernando Cedeño, llega a ser la película más distribuida en la historia cinematográfica del país, vendiendo un millón doscientas cincuenta mil copias según datos publicados por la Fundación Ecuatoriana de Cine Ocho y Medio.

Según la opinión de Ana $\mathrm{Cano}^{6}$, artista esmeraldeña, conocida artísticamente como Black Mama, integrante de "Somos mujeres. Somos Hip Hop" y cantante de géneros como el Soul, Jazz, Ragga y actualmente Hip Hop, la música "es un indicativo súper fuerte de la hipersexualización". En relación a los "narcocorridos" y el reggaetón afirma que lo que trasmiten estos géneros "pone en peligro la seguridad sexual de muchas niñas. Muchas baladas, esas corta venas, me suicidaré porque mi novio me dejó, ponen en peligro la vida de muchas adolescentes que lo consumen y en realidad no siempre es por falta de acceso de comunicación sino por el exceso de apoyo y exposición a este tipo de música".

La campaña colombiana "Usa la razón"7 (2014) hace visible la violencia en el reggaetón, concienciando sobre la violencia y el machismo de las letras de las canciones. Sin embargo, como se revela en el estudio "Músicas populares urbanas, relaciones de género y persistencia de prejuicios" (Hernández y Maia, 2013), muchas canciones deslumbran y atraen no por la atención de los y las oyentes en su letra si

\footnotetext{
${ }^{6}$ Opiniones recogidas en entrevista personal realizada en Quito, en marzo de 2015

7 La campaña "Usa la Razón" fue creada en Universidad Jorge Tadeo Lozano de Bogotá, Colombia. Caracterizada por su crudeza visual, la campaña se viralizó en redes sociales y apareció en medios de comunicación de la mayoría de países de América Latina y también en países europeos como Alemania, Francia, Estados Unidos y países de Asia.
} 
no por sus características rítmicas, melódicas y armónicas. El ritmo tiene que ver con los "latidos del corazón", de ahí su importancia para la artista Black Mama que señala en este sentido:

El ser humano todo el tiempo está en constante ritmo por los latidos del corazón y eso va a hacer que te identifiques con el tambor, por eso hasta el suizo "más arrítmico" llega acá a una noche de tambores y llega a bailar. Muchos géneros se han aprovechado de esta sensación del latido del corazón para crear, para provocar sensaciones. El reggaetón, -de los latidos del corazón- tiene unas cuantas micronésimas un poquito más aceleradas y lo que hace el cuerpo humano siempre va a ser adaptarse al entorno donde está. Entonces, si yo entro a una discoteca, así no me guste el reggaetón, empiezo a escucharlo y a bailar" (Entrevista personal, 24 de marzo de 2015)

\section{Imagen 1: Imagen de la campaña "Usa la razón"}

Fuente: Perfil de Facebook de la Campaña

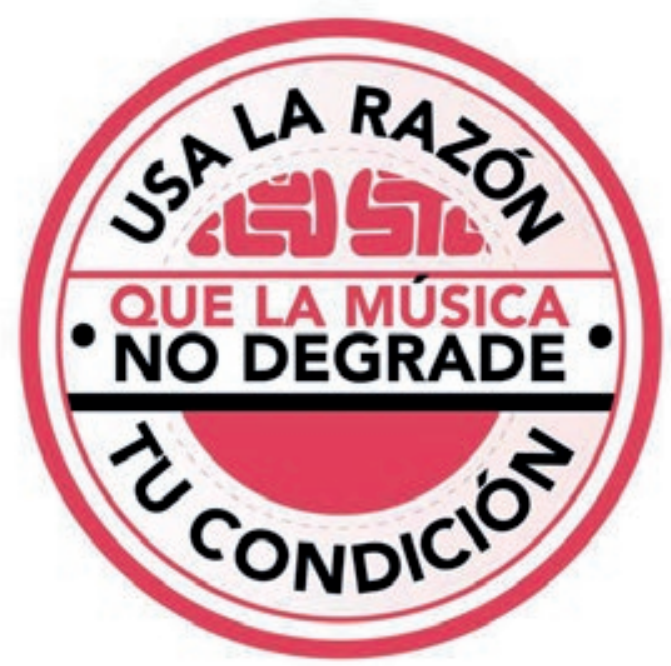

https://www.facebook.com/pages/Usa-la-raz\%C3\%B3n/276397465860330

El artículo "Música y sociedad en el capitalismo tardío" (Moench, 2011) se pregunta qué relación existe entre música y sociedad y si existen efectos políticos generados por la música. Las investigadoras y los investigadores de la asignatura Perspectiva de género, de la Facultad de Humanísticas y Ciencias Sociales de la Universidad Técnica de Manabí, nos hemos preguntado para esta investigación en qué medida el imaginario colectivo musical en Ecuador está influenciado por la violencia y el sexismo social.

Para el desarrollo de la investigación se realizó un contraste de hipótesis en dos muestras independientes. Por un lado, se analizaron los gustos musicales de 127 alumnas y alumnos que han cursado la asignatura Perspectiva de género; por otro, de 66 alumnas y alumnos que no han cursado la asignatura. La muestra total estuvo constituida por 193 alumnas y alumnos $(n=193)$ de la Universidad Técnica de Manabí, Ecuador. 


\subsection{La música que suena en Ecuador.}

La "diversidad cultural sonora de Ecuador" ${ }^{8}$ es sin duda un reflejo de la diversidad étnica y geográfica del país. El Instituto Nacional de Patrimonio Cultural, en el marco de la Convención de la UNESCO (2003), ha iniciado un registro del mundo sonoro en sus diferentes formas para poder tener un acercamiento a los gustos musicales del país.

En nuestro estudio, para lograr identificar los gustos musicales nacionales, optamos por realizar un análisis de emisoras de radio que poseen programas musicales o de variedades. Analizamos segmentos musicales durante los dos primeros meses del año 2015. El tiempo de sintonización de las radios fue distribuido en las diferentes programaciones y emisoras seleccionadas, lo que permitió un análisis de los programas desde una perspectiva de género.

\begin{tabular}{cc}
\hline RADIOS NACIONALES & RADIOS LOCALES \\
\hline Los 40 Principales & Radio Escandalo (Portoviejo) \\
Alfa Radio & Radio Amiga (Portoviejo) \\
Onda Cero & Radio Activa (Cuenca) \\
Joya Stereo & Radio Alfa Súper Stereo (Quito) \\
JC Radio La Bruja & Radio Farra (Portoviejo) \\
Radio La Luna (online) & Radio Modelo (Manta) \\
Radio Canela & Bahía Stereo (Bahía de Caráquez) \\
Radio La Rumbera & Radio diamante (Machala) \\
\hline Top Latino (online) & Radio Onda Positiva (Guayaquil) \\
Radio EXA & Punto Rojo (Guayaquil) \\
Radio Elegidos 45 (online) & Radio UNO (Durán / Guayaquil) \\
Radio Más Candela & \\
Radio Galaxia & \\
\hline
\end{tabular}

Tabla 1. Relación de emisoras de radio analizadas.

Fuente: Elaboración propia

El análisis radiofónico determina que los programas de radio de las emisoras sintonizadas son en su totalidad presentados por hombres salvo contadas excepciones como, el programa "Te cuento" de Radio Amiga, presentado por dos mujeres. Por lo tanto, la presencia de mujeres en la dirección de los programas musicales es minoritaria, reflejando segregación horizontal en el ámbito laboral de la comunicación en las radios del país.

En el caso de las audiencias, se observa una amplia representación de mujeres, salvo en el programa "A toda música" de los "Los 40 Principales" donde se identificó una

\footnotetext{
${ }^{8}$ Así lo denomina el Ministerio Coordinador de Patrimonio.
} 
inusual paridad, probablemente por la combinación de música electrónica y rock con baladas en inglés. En el caso de radio "La Rumbera" y su programación "En ritmo de bolero", la audiencia en su mayoría es masculina, considerando que esto puede deberse a que tanto el bolero como el pasillo se vinculan culturalmente al alcohol, los contenidos de las letras están relacionados con las decepciones amorosas y en el caso de Ecuador, por estar asociado a figuras artísticas como Julio Jaramillo que imprimen un fuerte carácter masculino en la construcción de la identidad cultural.

Se observa un gusto musical diferenciado en función de la clase social y del lugar geográfico. En lo que se refiere a la Región Costa, el género musical que predomina es el romántico en la Bachata, Balada, o el Pop, y en la Región Sierra, la Electrónica y el Rock.

Existe, a pesar de compartir la misma cultura latina, una gran preferencia por géneros musicales anglosajones y americanos dentro de la Región Sierra, más influenciada por la asimilación cultural de temas extranjeros que han opacado la música nacional como claro proceso de influencia norte americana y de la globalización y uso y difusión de la música a través de las nuevas tecnologías.

La música cumple una función importante en la construcción de roles y estereotipos. En el caso de las representaciones de las mujeres, destacan en las canciones más solicitadas en las radios del país, las que van desde los imaginarios estereotipados de la mujer sumisa, sufridora y tierna hasta las de las mujeres malas y putas, o en palabras de Virginia Guarinos "de la romántica a la mujer fálica" (Guarinos, 2012).

En las diferentes radios investigadas se puede constatar a través de las audiciones que las personas que más realizan llamadas telefónicas para solicitar canciones son en su mayoría hombres. Las mujeres, en cambio, envían más mensajes de texto. Sin embargo, la participación en redes sociales es equitativa.

Atendiendo a los grupos de edad, la juventud, en su mayoría, busca el género urbano o extranjero, y las personas de mayor edad, por lo general en todo el país, prefieren música Chichera, Tecnocumbia y románticas antiguas.

Como rasgo característico nacional, hay una tendencia a repetir de forma desmesurada la música en función de los sucesos ocurridos, optando por un tratamiento sensacionalista en el caso de la muerte de artistas, y también en fechas significativas como se pudo observar el 14 de Febrero, Día de San Valentín, conocido en el país como día del amor y la amistad, en donde las canciones románticas y de alto contenido sexista son las más solicitadas.

\subsection{Música y sexismo: la literatura musical desde una perspectiva de género.}

Los estudios de musicología feminista destacan en Estados Unidos y Canadá desde los años noventa con influencia del posmodernismo. Se denomina "música de género" o "musicología feminista" a la rama que estudia los aspectos de la musicología con la figura de las mujeres como eje central para visibilizar sus aportaciones a lo largo de la historia. La producción ha sido extensa en esta última 
década (Lorenzo Arribas, 1992; Piñeiro, 2000, 2001, 2008; Green, 2001; Campos, 2011; Ramos, 2003, 2010, 2013), con estudios también específicos sobre América Latina y el feminismo en la música (Piñeiro, 2011).

Según Patricia Digón Regueiro (2000) los estudios relacionados con el género y la música se pueden dividir en tres áreas de investigación: estudios sobre la historia de las mujeres en música; estudios sobre el género y el significado de la música; y estudios sobre género y educación musical (Zavala Girones, 2012; Martínez Escribano, 2011; Díaz Mohedo, 2005; Loizaga Cano, 2005; Viñuela Suárez, 2003; Berrocal de Luna y Gutiérrez Pérez, 2002). Sin embargo, esta clasificación expuesta años atrás por la autora se ha ampliado en estos diez años.

En relación a la importancia de la música en la cultura global y en como ésta es creadora y correctora de estereotipos de género destaca el estudio "El género de la música en la sociedad global" (Martínez Berriel, 2011). En Ecuador, a nivel local, se ha presentado recientemente una investigación de maestría en esta línea sobre la música global y su incidencia en la creación de géneros musicales ecuatorianos (Capa Plasencia, 2014).

En las investigaciones más recientes se incorpora el análisis de género en las propias letras de las canciones, determinando los roles y estereotipos asignados a hombres y mujeres (Martínez Moriega, 2014; Hernández Romero y Maia, 2013; Guarinos, 2012; Viñuela, 2011). El tratamiento de la violencia de género, directamente implícita en las canciones, se recoge en escasos estudios y muy locales (Velásquez- Barba, 2014)

A nivel nacional no se encuentran trabajos desde una perspectiva de género sobre estilos musicales ecuatorianos, solamente se registran investigaciones en relación a diferentes estilos musicales sobresalientes en el país como son el Pasillo (Granda, 2004), el Metal (Martínez, 2012) y la Tecnocumbia (Troya, 2008).

\section{OBJETIVOS}

Tal y como hemos planteado, la música es un fiel reflejo de la sociedad en la que vivimos, reproduciendo las desigualdades sociales y en las relaciones de género. Por lo tanto, el análisis de la misma nos ayuda a entender los mecanismos de reproducción, control y subordinación social.

El objetivo general ha sido determinar en qué medida la adquisición de la conciencia social de género influye en el imaginario musical.

Para ello, se han planteado los siguientes objetivos específicos:

1. Analizar de forma general los gustos musicales en Ecuador.

2. Analizar las canciones seleccionadas por las alumnas y los alumnos desde una perspectiva de género. 


\section{METODOLOGÍA}

La metodología utilizada en esta investigación ha consistido en el análisis de género de un total de 127 canciones seleccionadas por las alumnas y los alumnos de diferentes paralelos de la asignatura de Perspectiva de género de la Universidad Técnica de Manabí.

La investigación se ha realizado como parte de las actividades de investigación incluidas en el sílabo de la asignatura y para ello se han utilizado fuentes primarias y secundarias como la lectura de artículos científicos; la audición de emisoras de radio; la elección de la canción preferida por cada alumna y alumnos (al margen de que fuera sexista o no, violenta o no); el análisis de género teniendo en cuenta un análisis del lenguaje sexista y un análisis conceptual aplicado sobre la identificación de roles de género, estereotipos, prejuicios, elementos de amor romántico (celos, monogamia, heterosexismo, dependencia emocional...), violencia implícita y explícita (relaciones de dominación, control, superioridad, abuso, acoso, explotación...). Como conclusión, cada canción debía llevar una reflexión sobre la carga sexista y binaria. Este trabajo de identificación y análisis de género se realizó de forma individual y el posterior desarrollo del trabajo de manera colaborativa en grupos de investigación.

El análisis consistió en determinar de forma estadística: estilo musical de las canciones; número de autoras y autores seleccionados; países de origen de las canciones, época musical; y porcentajes de roles, estereotipos, elementos de amor romántico, prejuicios y violencia contenida en las canciones. El objetivo final fue determinar el porcentaje de sexismo en las canciones como una micro representación socio cultural de Ecuador.

Por otra parte, se ha contrastado la muestra con 66 alumnos y alumnas que no tienen noción alguna de perspectiva de género y que cursan estudios de Ciencias de la Educación pero con especialización en ciencia.

La muestra que al $100 \%$ ha cursado la asignatura de Perspectiva de género está representada por 127 personas, correspondientes a diferentes paralelos de las carreras de Psicología Clínica, Trabajo Social y Secretariado Ejecutivo de la Facultad de Humanísticas y Ciencias Sociales de la Universidad Técnica de Manabí. Por otro lado, se ha tomado una media muestral de 66 personas que corresponden a carreras de Ciencias de la Educación con especialización en ciencia y que no han cursado la asignatura de Perspectiva de género o de Equidad de género, las dos versiones que aparecen en las mallas curriculares de alguna de las carreras de la Universidad.

De la muestra de 127 personas que han cursado Perspectiva de género en el semestre 2014- 2015 se resuelve que el $80 \%$ son de sexo femenino y $20 \%$ masculino, lo que determina una muestra feminizada. El $63 \%$ son de origen urbano y el $37 \%$ de origen rural de la provincia de Manabí, Ecuador.

Los datos más destacados en el análisis muestral revelan que el $68 \%$ de las canciones elegidas son interpretadas por hombres -con autoría original también en un $91 \%$ de hombres-, el $26 \%$ por mujeres y el $6 \%$ por dúos mixtos. 
Entre los países de origen de la canción seleccionada destacan: México (31\%), EE.UU (16\%), Colombia (13\%), España (12\%), Puerto Rico (9\%) y Guatemala (6\%). Los temas elegidos están concentrados desde la década de los 90 hasta la década de los dos mil y en un $30 \%$ en la década del 2010.

Los estilos musicales que destacan son: la Balada (33\%), el Pop Latino (22\%), el Pop (15\%) y el Reggaeton (12\%).

Los motivos principales que motivan la elección de canción son en su mayoría: sentimientos de felicidad (32\%); "terapia frente a los problemas o sentimientos" (24\%); amor (18\%); y por sentirse identificado con la letra (12\%) o con el ritmo (11\%). Solo el $5 \%$ señalan ruptura amorosa como uno de los motivos de la elección y el $1 \%$ porque es una canción que le trasmite autonomía.

El $72 \%$ considera que la canción elegida es propia de su cultura y como motivos explican en un alto porcentaje, que transmite la realidad del país en un $17 \%$ (indicando entre otras cuestiones violencia, machismo, romanticismo, desamor, celos...) lo que legitima estas prácticas violentas como culturales. Otro 17\% también lo elige porque "es lo que se escucha en el país", y el $16 \%$ porque identifica el romanticismo como "algo propio del país y de la cultura latina".

El 69\% de las personas se consideran educadas en el amor romántico debido a la socialización primaria, especialmente, por lo que consideran "influencia de la cultura amorosa de su familia".

A la pregunta de si se considera una persona machista, escasamente el $19 \%$ considera que si, frente a un $81 \%$ que considera que no es machista. La mayoría justifica que no es una persona machista, argumentando "que no les gusta imponer" y que "creen en una sociedad igualitaria".

En cuanto al análisis de las canciones, el 59\% reconoce que la canción es sexista, señalando altos porcentajes de cuestiones de género: construcciones de amor romántico $(27 \%)$; roles $(24 \%)$; estereotipos $(18 \%)$; prejuicios $(13 \%)$; binarismo social $(10 \%)$ y discriminación en las letras de la canción (7\%). 


\section{ANÁLISIS DE GÉNERO DE LA CANCIÓN SELECCIONADA}

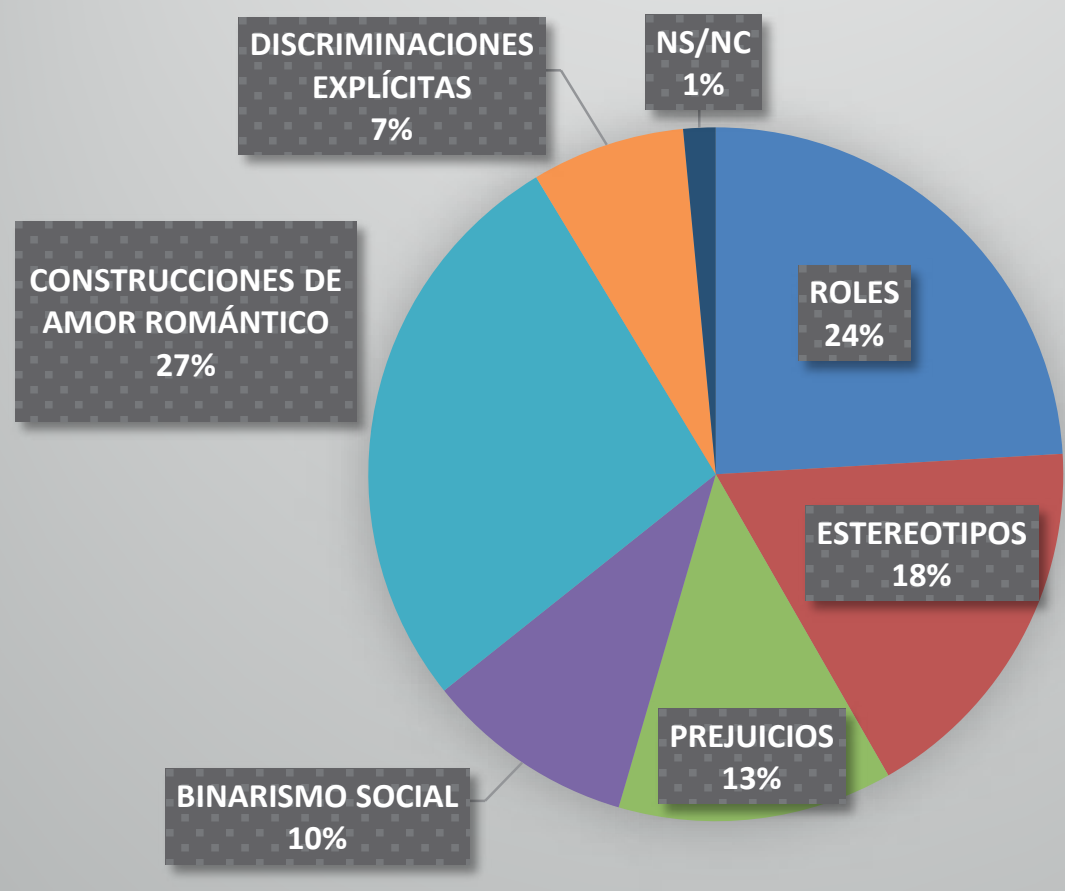

Gráfico 1: Elementos del análisis de género identificados por las alumnas y los alumnos que han cursado la asignatura de Perspectiva de género.

Fuente: Análisis de datos de la investigación

Es interesante contrastar estos datos, con los resultados obtenidos en la muestra de las sesenta y seis (66) estudiantes que no han cursado la asignatura Perspectiva de Género. De esta muestra reducida, el $41 \%$ eran hombres y el $59 \%$ mujeres, lo que supone una muestra nuevamente feminizada aunque en menor proporción que la analizada con anterioridad.

El 79\% eligió un intérprete masculino y solo el $18 \%$ femenino -con autoría también masculina en un $77 \%$ de los casos-. Entre los países en donde los artistas han desarrollado sus carreras profesionales, se encuentran: Guatemala (25\%); Puerto Rico (17\%); EE.UU (15\%) o España (8\%), entre otros. Los estilos musicales destacados son la Balada Romántica (33\%) y el Pop (21\%).

La publicación de las canciones se concentra desde los años 90 hasta la actualidad. Los principales motivos por los que eligen las canciones son porque se sienten identificados con la letra (17\%) y por sentimientos de felicidad (23\%). 
Reconocen que las canciones elegidas no son propias de su cultura en un 50\% pero consideran que forman parte del imaginario romántico, aunque en la mayoría de los casos existe una dificultad para tal identificación.

El $82 \%$ consideran estar educados en el amor romántico pero la mayoría no saben determinar por qué motivos y solo el $7 \%$ define entre los motivos la educación. Sin embargo, el $89 \%$, casi la misma proporción, no se considera una persona machista argumentando entre otras cuestiones porque "cree en la igualdad de los sexos".

El $51 \%$ identifica en la canción elegida construcciones de amor romántico y el $26 \%$ roles de género. Pero a la pregunta si consideran que su canción es una canción sexista, el $86 \%$ considera que no y un escaso $12 \%$ que sí.

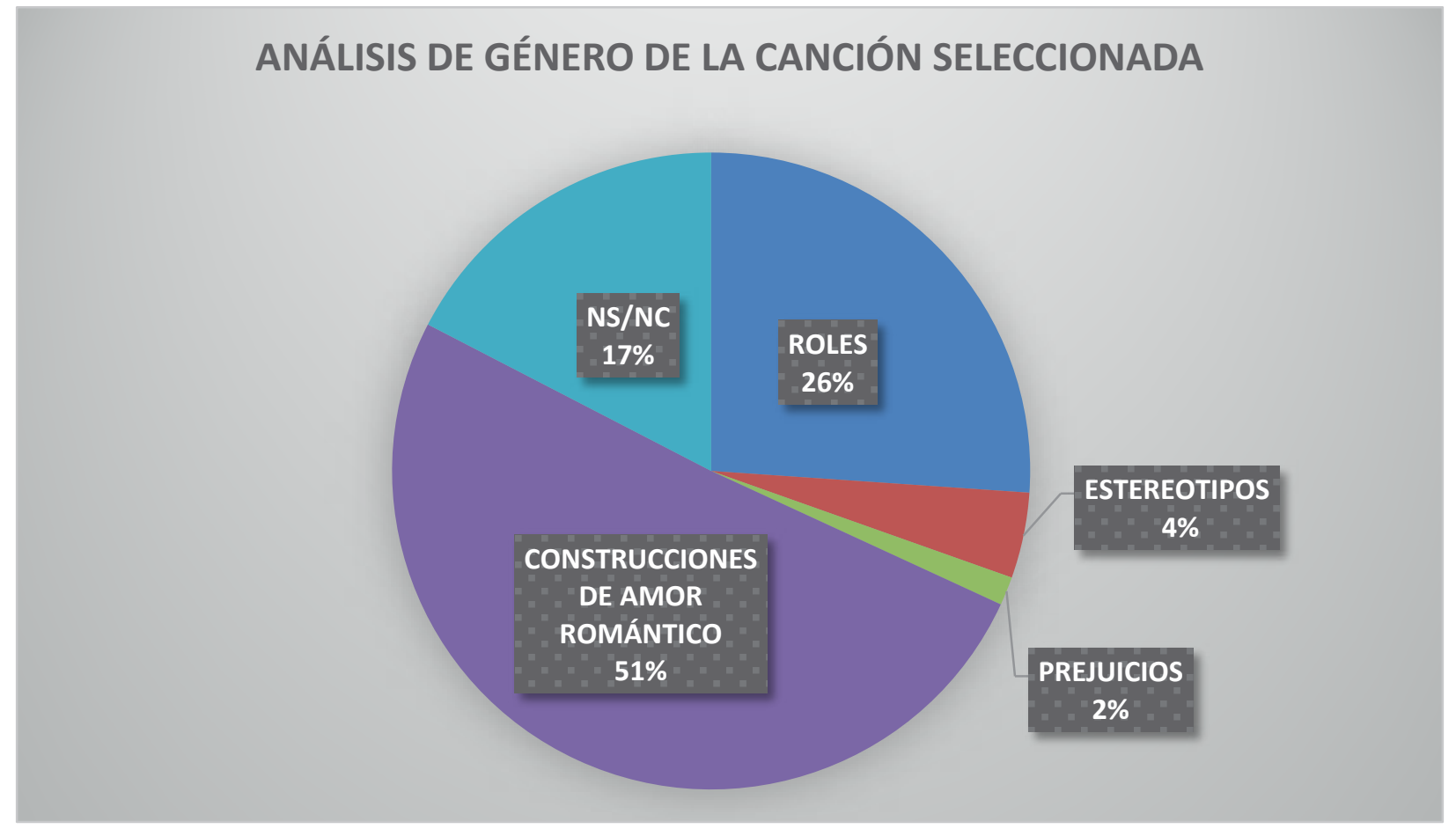

Gráfico 1: Elementos del análisis de género identificados por las alumnas y los alumnos que no han cursado la asignatura de Perspectiva de género.

Fuente: Análisis de datos de la investigación

\section{DISCUSIÓN}

La metodología empleada de investigación participante ha podido determinar que la conciencia de género y el análisis crítico de la realidad social es una asignatura aún pendiente en las sociedades contemporáneas, incluso en las generaciones más jóvenes.

Del estudio se pueden extraer algunos debates importantes que también lo han sido para la Teoría Feminista. El análisis musical de la investigación, desde una 
perspectiva de género, revela fuertes construcciones del imaginario de amor romántico en hombres y mujeres, aunque en mayor medida en mujeres al ser una muestra feminizada. Lo que determina que su vigencia es persistente, a pesar de ser un modelo sociocultural de amor del siglo XIX, fruto de una construcción económica, social y cultural patriarcal, basada en el desigual reparto de papeles para hombres y mujeres.

En el estudio se puede comprobar como la mayor parte de las personas participantes en la muestra se consideran educadas en el mito del amor romántico y lo asumen en sus relaciones con normalidad, fruto de su educación y socialización familiar. Además, como consecuencia de un proceso de modernización, en el que la construcción de amor romántico tradicional se ha vaciado de los perjuicios ocasionados a lo largo de la historia para hombres y mujeres e idealizado por los medios de comunicación bajo la idea del "amor ideal", y por el capital, en donde el amor se vuelve también consumo.

No es de extrañar por tanto, que los estilos predominantes en nuestra investigación sean la Balada Romántica, el Pop Latino o el Reggaeton, aunque éste último en menor proporción. En este punto es conveniente hacer un análisis, no solo en relación a los gustos musicales sino un análisis social y de género de los lugares de la producción musical.

En relación al estilo musical se identifica claramente que los estilos clásicos de música -al igual que el modelo de amor tradicional- persisten en la juventud aunque se han abandonado otros estilos clásicos nacionales como por ejemplo, El Pasillo. Estilos como la Balada Romántica y el Pop Latino son los favoritos y los representantes de letras de alto contenido sexista. La Balada Romántica o Balada Latina surge en España y deriva directamente de La Copla, un estilo cantado principalmente por mujeres con fuertes marcadores de identidad en el mantenimiento de una cultura machista y estereotipos de feminidad y masculinidad que generan relaciones de subordinación.

En cuanto a la producción de música de alto contenido sexista proviene en altos porcentajes de países como México, EE.UU, Colombia, España o Guatemala. Un país como es EE.UU. representante del capitalismo y las desigualdades sociales, o países como el resto de los mencionados que destacan por registrar altos índices de violencia de género, femicidio y feminicidio. Por ejemplo, en el caso de Guatemala, que ha registrado más de cinco mil muertes violentas de mujeres en una década, según datos de la página Web "Femicidio.net" y donde los índices de impunidad alcanzan el 98\%, según la Comisión Internacional contra la Impunidad de Guatemala. En países con estos datos es donde se producen la mayor parte de las supuestas "canciones de amor". Y es inevitable aquí revindicar la consigna "el amor no es violencia".

De esta reflexión se deduce que es necesario seguir insistiendo en el hecho de que el amor romántico en el que se educa a la mayor parte de la población, perpetúa los mecanismos de sometimiento y subordinación y, como podemos ver en este estudio, la industria musical, es un mecanismo en alianza con la producción de las relaciones de desigualdad en el mundo; dejando a la luz nuevamente, la perversa vinculación entre el capital, el poder y las condiciones de desigualdad para mujeres y hombres. 
Siguiendo con los resultados de nuestro estudio, las alumnas y los alumnos, a pesar de considerar en altos porcentajes que han recibido una educación basada en el imaginario del amor romántico y por tanto, en relaciones de desigualdad, no se consideran en altos porcentajes personas machistas, sin existir diferencias significativas entre la muestra que ha cursado asignatura de género y la que no lo ha cursado. Ambos grupos se consideran "románticos y románticas" pero no logran identificar los mecanismos de subordinación del amor romántico con el machismo, el sexismo o la violencia.

Lo que sí logran en una mayor proporción las alumnas y los alumnos que han cursado la asignatura de género, es señalar en la canción elegida un mayor número de cuestiones de género, destacando la identificación de construcciones de amor romántico, roles y estereotipos. Lo que nos lleva a concluir que, pese a que son identificadas y señaladas las desigualdades, las personas se siguen identificando con los mecanismos de la desigualdad y concretamente en nuestro estudio, con las letras de estas canciones que les siguen produciendo sentimientos como se señala entre otros, de "felicidad". Por lo tanto, existe una reproducción y tolerancia social, como resultado de una débil conciencia de género.

Sí hay una diferencia en los grupos estudiados sobre la consideración del sexismo en la canción elegida, siendo el grupo que ha cursado la asignatura de género, el que concluye en mayor proporción en el análisis que su canción es sexista.

\section{CONCLUSIONES}

A modo de conclusión de nuestra investigación, podemos determinar que la adquisición de la conciencia social de género influye débilmente en el imaginario musical ya que persisten elementos de una socialización desigual para hombres y mujeres desde edades muy tempranas, y es difícil su modificación a través de la adquisición reciente de las nociones de género.

A partir de aquí, el reto será seguir apoyando en el ámbito educativo una formación de calidad basada en valores como la equidad, la inclusión, la diversidad y la igualdad, generando cuerpos teóricos y prácticos con legitimidad académica que permitan no sólo la identificación de las desigualdades sociales sino el compromiso de su erradicación y un pacto de Estado basado prioritariamente en la justicia social y de género.

\section{REFERENCIAS}

\section{PUBLICACIONES PERIÓDICAS, REVISTAS, WEBES Y SIMILARES:}

Altamirano, Magdalena (2010). Representaciones femeninas en el corrido mexicano tradicional: heroínas y antihéroes. Revista de Dialectología y Tradiciones Populares, Vol. 65, (2), pp. 445-464 
Berrocal de Luna, Emilio \& Gutiérrez Pérez, José (2002). Los roles sociales y el género en las canciones populares. Eufonía: Didáctica de la música, (25), pp. 100108

Berrocal de Luna, Emilio \& Gutiérrez Pérez, José (2002). Música y género: análisis de una muestra de canciones populares. Comunicar: Revista científica Iberoamericana de comunicación y educación, (18), pp. 187-190

Bialowas Pobutsky, Aldona (2011). Deleitar denunciando. La narco telenovela de Gustavo Bolívar "Sin tetas no hay paraíso" marca el pulso de la sociedad colombiana". Espéculo: Revista de Estudios Literarios, (46)

Callejo, Javier (2011). Reseña de "La muerte como espectáculo" de Michela Marzano. Empiria: Revista de Metodología de las Ciencias Sociales, (22), pp. 214-215

Campos, Susana (2011). La musicología feminista ante el Plan de Educación para todos. Música y Educación, (85), pp. 216-224

Díaz Mohedo, María Teresa (2005). La perspectiva de género en la formación del profesorado de música. REICE: Revista Electrónica Iberoamericana sobre Calidad, Eficacia y Cambio en Educación. Vol. 3, (1), pp. 570-577

Digón Regueiro, Patricia (2000). Género y Música. Música y Educación: revista trimestral de pedagogía musical, Vol. 13, 1, (41) pp. 29-54

García, Alexander (6 de enero de 2015). El pueblo de Durán salió a despedir a Sharon, su diva. El Comercio. Recuperado de http://www.elcomercio.com/tendencias/duran-salio-despedir-sharon.html

García, Alexander (6 de enero de 2015). Una multitud le dijo adiós a la 'Diva' con su Tecnocumbia. El Comercio. Recuperado de http://www.elcomercio.com/tendencias/sharon-multitud-sepelio-divafunerales.html

Segato, Rita (10 de abril de 2015). Entrevista Facultad de Periodismo y Comunicación Social. Universidad de Costa Rica. Recuperado de http://perio.unlp.edu.ar/node/4602

Granda, Wilma (2004). El pasillo ecuatoriano: noción de identidad sonora. Iconos: Revista de Ciencias Sociales, (18), pp. 63-70.

Guarinos, Virginia (2012). Estereotipos y nuevos perfiles de mujer en la canción de consumo. De la romántica a la mujer fálica. Cuestiones de género: de la igualdad y la diferencia, (7), pp. 297-314 
Hernández, Nieves; Maia, Ari F (2013). Músicas populares urbanas, relaciones de género y persistencia de prejuicios. Análisis de la comprensión de seis canciones por jóvenes españoles y brasileños. Musiker. cuadernos de música (20), pp. 207253

Hernández Romero, Nieves \& Ari Fernando Maia, Ari (2013). Músicas populares urbanas, relaciones de género y persistencia de prejuicios: análisis de la comprensión de seis canciones por jóvenes españoles y brasileños. Musiker: cuadernos de música, (20), pp. 207-253

Loizaga Cano, María (2005). Los estudios de Género en la Educación Musical. Revisión Crítica. Musiker: cuadernos de música, (14), pp. 159-172

Lorenzo Arribas, Josemi (1992). Musicología feminista Medieval. Cuadernos de Investigación Medieval, (11), pp. 9-109

Martínez Berriel, Sagrario (2011). El género de la música en la cultura global. Trans: Revista Transcultural de Música, (15), pp. 1-17

Martínez Escribano, Luis Miguel (2011). Sexismo en la música pop española. Logros y retos: Actas de III Congreso universitario nacional Investigación y género. Unidad de Igualdad de la Universidad de Sevilla, España, pp. 1214-1223

Martínez Noriega, Dulce Asela (2014). Música, imagen y sexualidad: el reggaetón y las asimetrías de género. El Cotidiano, (186). Universidad Autónoma Metropolitana Unidad Azcapotzalco Distrito Federal, México, pp. 63-67

Moench, Eric (2011). Música y sociedad en el capitalismo tardío. Nómadas. Revista Crítica de Ciencias Sociales y Jurídicas Vol. 31, (3)

Piñeiro Gil, Carmen Cecilia (2001). Los estudios de género en la música. Universidad de Oviedo. España.

Piñeiro Gil, Carmen Cecilia (2011). Latinoamérica y el feminismo en música: caminos recorridos. Mujer versus Música: itinerancias, incertidumbres y lunas. pp. 37-76

Piñeiro Gil, Carmen Cecilia (2008). Música y Mujeres, género y poder: diez años después. Itamar: Revista de investigación musical: territorios para el arte, (1), pp. 201-211

Ramos, Pilar (2013). Una historia particular de la música: la contribución de las mujeres. Revista Brocar: Cuadernos de investigación histórica (37), pp. 207-224.

Ramos, Pilar (2010). Luces y sombras en los estudios sobre las mujeres y la música, Revista Musical Chilena, Vol. 64, (213), pp. 2-25 
Santillán, Alfredo \& Ramírez, Jacques (2004). Consumos culturales urbanos: el caso de la Tecnocumbia en Quito. Íconos. Revista de Ciencias Sociales (18), pp. 43-52.

Velázquez- Barba, Rosario Eliud (2014). Vulnerar los espacios femeninos: suavizar la violencia a través de la canción mexicana y su difusión radiofónica. Ra Ximhai, Vol. 10. (7), pp. 71-82

Viñuela, Eduardo (2011). La subversión de los roles de género en la música popular: Mónica Naranjo como artista inapropiada/ble. Trans: Revista Transcultural de Música, (15) pp. 1-20

Viñuela Suárez, Laura (2011). La construcción de las identidades de género en la música popular. Dossiers Feministes. No me arrepiento de nada: Música y Mujeres, (7), pp. 11-33

Zavala Girones, Mercedes (2012). Música y género, en Suárez Ojeda, Magdalena (Coord.), Género y mujer desde una perspectiva multidisciplinar (pp. 121-138). Madrid: Editorial Fundamentos.

\section{LIBROS, CAPÍTULOS DE LIBRO O ENTRADA DE UN LIBRO DE CONSULTA, INFORMES TÉCNICOS, TESIS:}

Capa Plascencia, Meri Jhaned (2014). La música global y su incidencia en la creación de géneros musicales ecuatorianos en la asociación de artistas profesionales de la ciudad de Loja. (Tesis de Maestría en Pedagogía e Investigación musical). Universidad de Cuenca, Ecuador.

Código Orgánico Integral Penal, COIP (2014). Quito. Ecuador: Ministerio de Justicia, Derechos Humanos y Cultos.

Consejo Nacional de la Niñez y Adolescencia de Ecuador, CNNA. (s.f.). Estudio del Frente Ecuatoriano de defensa de los derechos sexuales y derechos reproductivos ¿Conoce usted la realidad de la violencia sexual en el Ecuador? Quito, Ecuador: CNNA

Green, Lucy (2001). Música, género y educación. Madrid: Morata.

Instituto Nacional de Estadística y Censos, INEC (2012). Encuesta Nacional de Relaciones Familiares y Violencia de Género contra las mujeres. Quito. Ecuador: INEC

Martínez Moreno, Inés (2012). Culturas urbanas. Los Black Metaleros en Quito. Concepciones de la familia y de la sexualidad. (Tesis de Maestría. Programa de Maestría en Estudios de la Cultura). Universidad Andina Simón Bolívar, Ecuador.

Ramos, Pilar (2003). Feminismo y música. Introducción crítica. Madrid: Narcea. Troya Molina, David (2008). Las estéticas lúdicas de la Tecnocumbia. (Tesis de Maestría. Programa de Maestría en Estudios de la Cultura). Universidad Andina Simón Bolívar. Ecuador. 
UNESCO (2003). Convención para la salvaguardia del patrimonio cultural inmaterial. París.

UNODC, Oficina de las Naciones Unidas contra la Droga y el Delito (2012). Informe Mundial sobre las Drogas. Nueva York.

\section{AUTOR/ES:}

\section{Cristina Mateos Casado}

Trabajadora Social por la Universidad de Salamanca (España). Socióloga y doctorado en Teoría Feminista por la Universidad Complutense de Madrid. Docente investigadora de la Facultad de Filosofía, Letras y Ciencias de la Educación de la Universidad Técnica de Manabí (Ecuador).

\section{María Pita Asan}

Ingeniera Civil. Analista en Sistema y Máster en Proyectos Educativos y Sociales. Docente investigadora de la Facultad de Filosofía, Letras y Ciencias de la Educación de la Universidad Técnica de Manabí (Ecuador).

\section{Mónica Vélez Zambrano}

Alumna de $5^{0}$ nivel de la Carrera de Trabajo Social de la Facultad de Ciencias Humanísticas y Sociales de la Universidad Técnica de Manabí (Ecuador).

Robert Cedeño Mejía. Alumno de $6^{\circ}$ nivel de la Carrera de Psicología Clínica de la Facultad de Ciencias Humanísticas y Sociales de la Universidad Técnica de Manabí (Ecuador).

José Ruíz Villamar, alumno de $5^{\circ}$ nivel de la Carrera de Bibliotecología y Ciencias de la Información de la Facultad de Ciencias Humanísticas y Sociales de la Universidad Técnica de Manabí (Ecuador). 